\title{
Article \\ Intra Prediction-Based Hologram Phase Component Coding Using Modified Phase Unwrapping
}

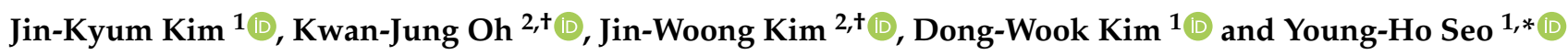 \\ 1 Department of Electronic Materials Engineering, Kwangwoon University, Seoul 01897, Korea; \\ jkkim@kw.ac.kr (J.-K.K.); dwkim@kw.ac.kr (D.-W.K.) \\ 2 Electronics and Telecommunications Research Institute, Daejeon 34129, Korea; kjoh@etri.re.kr (K.-J.O.); \\ jwkim@etri.re.kr (J.-W.K.) \\ * Correspondence: yhseo@kw.ac.kr; Tel.: +82-2-940-8362 \\ + These authors contributed equally to this work.
}

check for updates

Citation: Kim, J.-K.; Oh, K.-J.; Kim, J.-W.; Kim, D.-W.; Seo, Y.-H. Intra Prediction-Based Hologram Phase Component Coding Using Modified Phase Unwrapping. Appl. Sci. 2021, 11, 2194. https://doi.org/10.3390/ app11052194

Academic Editor: Ting-Chung Poon

Received: 31 December 2020

Accepted: 23 February 2021

Published: 3 March 2021

Publisher's Note: MDPI stays neutral with regard to jurisdictional claims in published maps and institutional affiliations.

Copyright: (c) 2021 by the authors. Licensee MDPI, Basel, Switzerland. This article is an open access article distributed under the terms and conditions of the Creative Commons Attribution (CC BY) license (https:// creativecommons.org/licenses/by/ $4.0 /)$.

\begin{abstract}
In this paper, we propose a method for compressing the phase component of a full-complex hologram. The JPEG (Joint Photographic Experts Group) Pleno is undergoing standardization for compressing full-complex holograms. If the full-complex hologram is compressed in the form of amplitude and phase components, the three-dimensional information of the hologram may be better preserved. Therefore, in order to solve the disadvantages of the method of independently compressing real and imaginary parts, we propose a method for directly compressing phase information. We select the HEVC (High Efficiency Video Coding), which has the best performance in compressing holograms from previous studies, as the anchor codec, and propose an algorithm for converting the phase information into the form suitable for the HEVC. Since the phase component is very random, we propose a modified phase unwrapping technique to improve this. In addition, in order to make good use of the property of HEVC Intra coding, the phase unwrapping considering Intra prediction is applied, and the most suitable HEVC Intra coding condition is searched. Compared with the result of compressing the phase using the HEVC, the hologram was improved by $2 \mathrm{~dB}$ or more and the reconstruction result was improved by more than $4 \mathrm{~dB}$ at a compression ratio of $80: 1$. If the compression ratio is increased, the proposed method has better results.
\end{abstract}

Keywords: hologram compression; phase unwrapping; HEVC Intra prediction; full-complex hologram

\section{Introduction}

The holography was first proposed by Gabor in 1948 [1] and has been researched and developed in many fields due to its ability to fully record 3D information. The analog holography is a technology that records 3D information on a holographic film made of a special material and develops the film. It is a somewhat limited technology for modern multimedia services [2]. Recently, studies on digital hologram technology have been widely conducted to fully utilize the three-dimensional reconstruction of holograms while overcoming the disadvantages of the analog method [3]. In order to use digital hologram as multimedia, digital hologram signal processing technology is required [4]. The hologram signal processing is largely composed of hologram rendering and compression. Hologram rendering techniques can include hologram creation, editing, display, interpolation, and enhancement. Hologram compression techniques include static hologram compression and video hologram compression. Currently, JPEG Pleno is in the process of standardizing compression for still holograms [5]. We will first briefly discuss various hologram compression methods that have been proposed or tried before.

The easiest and simplest hologram compression method to be applied would be the lossless coding method. In previous studies, several attempts have been made to compress holograms with a lossless method [6,7]. A hologram in the form of a complex number expressed in a floating-point format may be expressed as a pair of real (RE) and 
imaginary numbers (IM), or amplitude (AM) and phase (PH). Since these pairs display the same information, but show different characteristics, there is a possibility that the effect on compression will be different. In the previous study, the compression effect of the hologram did not appear in the case of the AM/PH pair, and the compression ratio of about 3:1 4:1 was shown in the case of the RE/IM pair [6].

A compression method using hologram resampling was also tried [8]. The classical form of resampling studies have been mainly conducted before [6], and because of the low compression ratio, few studies have been published recently. In terms of research results, the bilinear interpolation was the best result, followed by the bicubic interpolation, and finally the nearest neighborhood interpolation method [6].

Research that analyzed the effect of quantization on holograms can be seen in several research cases so far. In the past, uniform quantization was mainly used, and scalar quantization, which was considered in units of hologram pixels, was mainly considered [6]. Recently, research on vector quantization that performs quantization in block units is also being conducted [9]. In addition, scalar quantization and a uniform quantizer as well as a non-uniform quantizer were studied. Looking at the research results using normalized standard deviation (NSTD), the uniform quantizer shows good performance in the case of assigning low quantization bits (high compression), and the K-medians clustering method in the case of assigning high quantization bits (low compression) showed good performance. However, the difference is very small. Overall, the K-means clustering method showed low performance for all quantization bits. The results of this study show that vector quantizers with high computational complexity and complex algorithms do not necessarily show good performance for quantization of holograms.

Recently, various studies have been carried out to compress holograms using standard codecs such as JPEG, JPEG2000, AVC, and HEVC, and benchmarking the compression efficiency between them [10-12]. There are several common considerations in the way standard codec is used. The first is to convert a complex hologram in the form of floatingpoint into 8-bit integer data for input to the standard codec. The second is to adjust the parameters of the codec. Each codec has various parameters that can be adjusted. For example, in the case of HEVC, if the Intra mode is used, QP and CU size can be selected. In this way, some results may differ due to the difference in the setting of the preferred parameter.

Research on compressing a hologram can be classified into a method of compressing the hologram itself and a method of compressing the information of the surface diffracted (restored) at the position after propagating the hologram over a certain distance [13-16]. Most of the methods discussed so far have been attempted to compress the hologram itself. Since the hologram has a shape similar to noise, it is very difficult to find a spatial correlation between pixels, so it is difficult to show a good energy concentration even if a frequency transform is performed. Therefore, the compression method in the reconstruction domain is to compress the hologram after transforming it into a form of data with higher spatial correlation. We focus on the study of compressing the hologram itself.

Holograms can be expressed in various ways. If the hologram was photographed using a CCD by optical method, it would correspond to an intensity-based hologram, and in the case of a phase-shifted hologram, the difference between each hologram (Phase-shifted distances-based representation) is used for compression. The most common hologram is expressed as a complex object wavefield-based representation. Complex waves can be expressed as real and imaginary numbers, or as amplitude and phase. If information on the same hologram is represented by different expression methods, the result of compression may be different. In general, the real and imaginary representation shows better compression efficiency than the amplitude and phase pair. This is because the phase component has too strong randomness and the compression efficiency is very low. Therefore, most previous studies prefer to compress real and imaginary information. We propose a new method for compressing phase information. The proposed method assumes the use of HEVC as an anchor codec, and proposes a method for efficiently compressing the phase information of 
a complex hologram using a phase unwrapping technique [17] and a prediction mode of HEVC Intra.

This paper is organized as follows. Section 2 discusses the characteristics of the phase information of a full-complex hologram. Section 3 describes the proposed phase component compression method. Section 4 shows the experimental results, and Section 5 concludes this paper.

\section{Phase Information of Full-Complex Hologram}

This section describes the characteristics of the phase component in a full-complex hologram. We introduce phase unwrapping to improve the random characteristics of phase components, and analyze the effect of phase unwrapping on compression through experiments. We propose in Section 3 an algorithm for efficiently compressing the phase component based on this analysis result. In other words, through this section, we explain the basic motive of the technique through the experimental results.

\subsection{Effect of Phase}

As described above, a full-complex hologram is composed of complex numbers of real and imaginary parts. It can also be expressed in terms of amplitude and phase. We show the full-complex hologram in Figure 1. Figure 1a,b are real and imaginary components, respectively, and Figure 1c,d are amplitude and phase components. Figure 1e,f are the amplitude and phase components of the hologram diffraction result. In the case of compression, it is true that the real and imaginary components show better efficiency. However, when the real and imaginary components are independently compressed, the phase information of the hologram may not be considered, and the quality of the reconstruction result of the hologram may not be proportional to the quality of the hologram. The hologram, Horse in Figure 1, was optically captured using the phase-shifting method [18], and is published as an experimental data set in JPEG Pleno.

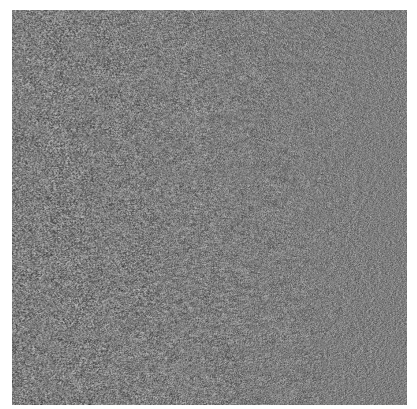

(a)

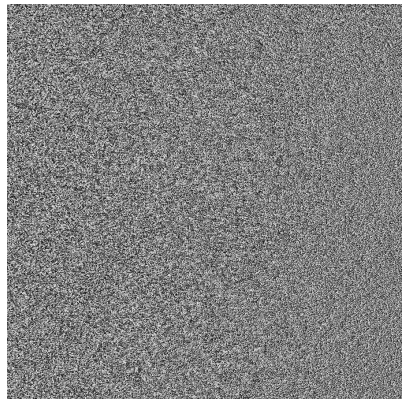

(d)

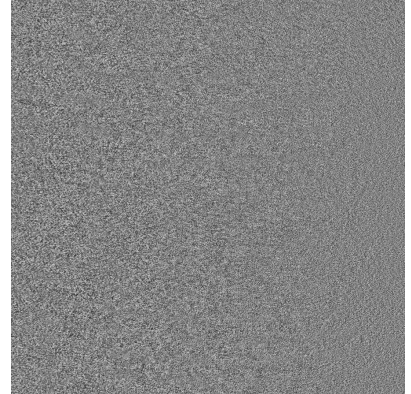

(b)

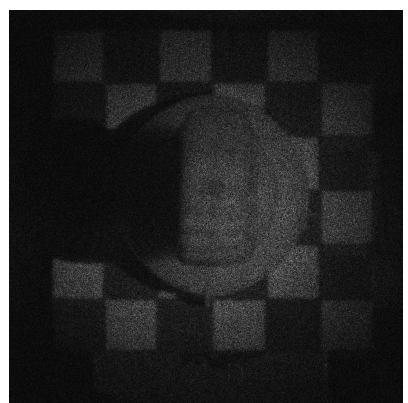

(e)

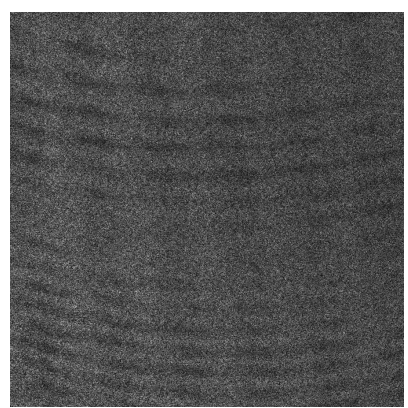

(c)

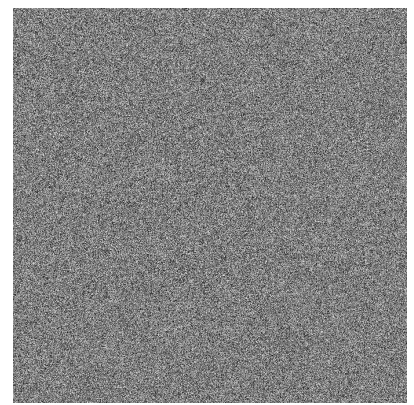

(f)

Figure 1. Components of full-complex hologram (Horse [18]): (a) real, (b) imaginary, (c) amplitude, and (d) phase of a hologram, (e) amplitude, and (f) phase of reconstruction. 
We tried to infer the characteristics of the phase component of the hologram by compressing the real and imaginary components in different ways. Reconstruction quality in four cases was considered according to the quality of hologram and phase as shown in Table 1. What we can note in this experiment is that even though the PSNR (31.54 dB) of the hologram is high, the quality of the reconstruction $(25.81 \mathrm{~dB})$ is not high when the PSNR $(16.68 \mathrm{~dB})$ of the phase is low. In comparison, the PSNR $(29.31 \mathrm{~dB})$ of the hologram is lower, and the PSNR $(26.43 \mathrm{~dB})$ of the reconstruction is higher when the PSNR of the phase is higher (17.11 dB). Through this experiment, we can confirm that not only the quality of the hologram itself, but also the phase information of the complex hologram are very important to the reconstruction efficiency. The phase information can be proportional to the consistency of the relationship between real and imaginary components. Compressing the phase information itself would be a very good way to maintain the phase relationship well. However, the phase information is very difficult to compress due to its randomness. Therefore, we try to efficiently compress phase information that is very difficult to compress.

Table 1. Relationship between phase information of holograms and the reconstruction result.

\begin{tabular}{cccccc}
\hline \multicolumn{3}{c}{ PSNR (dB) } & & SSIM \\
\hline Real & Imaginary & Complex & Phase & \multicolumn{2}{c}{ Reconstruction } \\
\hline 27.43 & 25.27 & 26.35 & 16.39 & 23.29 & 0.82 \\
31.43 & 27.18 & 29.31 & 17.11 & 26.43 & 0.90 \\
31.71 & 31.71 & 31.71 & 16.99 & 26.83 & 0.91 \\
31.78 & 31.29 & 31.54 & 16.68 & 25.81 & 0.87 \\
\hline
\end{tabular}

\subsection{Phase Unwrapping}

If the delay in the phase response can be expressed as a multiple of the sinusoidal period, we can unwrap this phase response. The distinction between a true phase delay and a phase delay with discarded sinusoidal periods is the most important factor for successful phase unwrapping. The purpose of this research is not to find the original phase response for phase unwrapping, but to increase the compression efficiency by making phase linearity, so the difficulty of such distinction is not a big problem. Figure 2 shows an example of phase unwrapping. As shown in Figure 2a, the phase, which was limited to a value from $-\pi$ to $\pi$, was expanded to a range of $-3 \pi$ to $6 \pi$ as shown in Figure $2 b$ through phase unwrapping. As a result, the phase has a linear shape. If we take a modulo operation with a value of 2 in the phase of Figure 2b, it becomes the same value as Figure 2a. We call this process rewrapping.

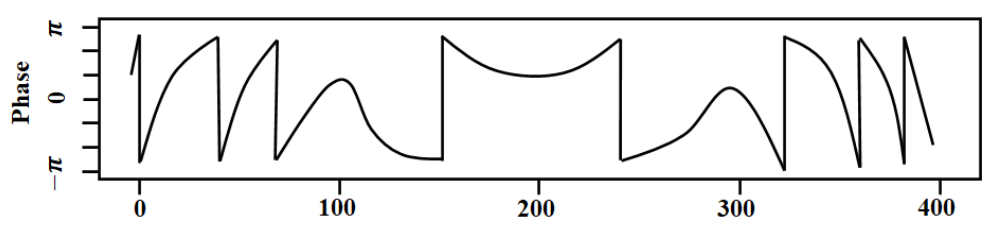

(a)

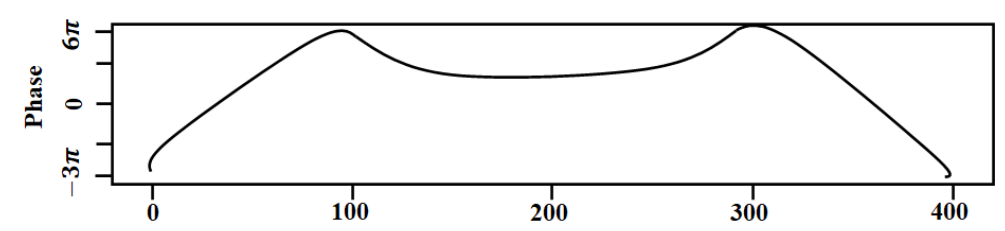

(b)

Figure 2. Example of phase unwrapping: (a) original phase response, (b) unwrapped phase response. 
Figure 3 shows the results of phase unwrapping for the phase component of a fullcomplex hologram. The unwrapping result of the original phase information in Figure $3 a$ is the image in Figure $3 \mathrm{~b}$. Figure $3 \mathrm{c}, \mathrm{d}$ are the results of plotting one row in the middle position. It can be seen that the phase changed very linearly after phase unwrapping. However, as described above, since the dynamic range is very wide due to phase unwrapping, when the result of phase unwrapping is normalized to a limited input width, the error per bit of the phase becomes very large.

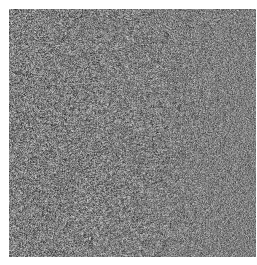

(a)

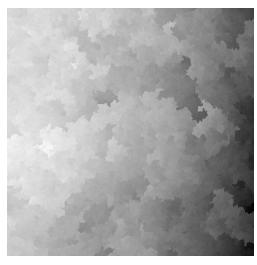

(b)

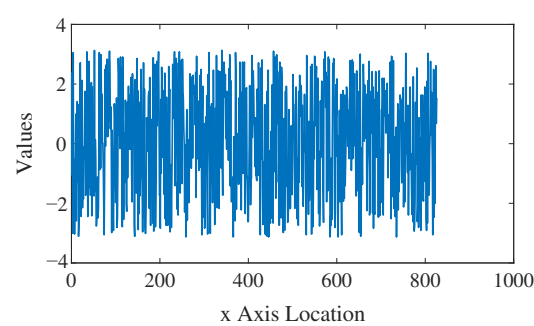

(c)

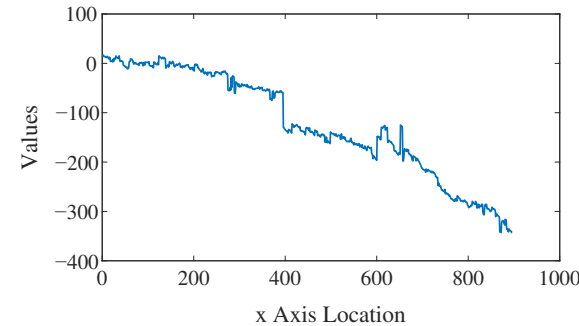

(d)

Figure 3. Effect of phase unwrapping: (a) original phase component, (b) unwrapped phase, (c) distribution of the original phase in a row, (d) distribution of the unwrapped phase in a row.

We consider the case of compressing a hologram using a standard codec (assuming the most common 8-bit input). If the original hologram phase component with a range of $2 \pi$ is normalized to 8 -bit integer type, the error has $2 \pi / 256$ per 1 bit. However, if the range is $160 \pi$ due to phase unwrapping and is normalized to 8 bits, the error is $160 \pi / 256$. Next, if data with such an error rate are compressed, the error per bit becomes too large and phase unwrapping acts as a disadvantage.

An experiment was performed on the effect of phase unwrapping using the Horse hologram [18] of JPEG Pleno. Figure 4 shows the PSNR value of the compression result while compressing up to 100:1 using the HEVC codec. It can be seen that almost similar compression trends are shown for all cases, and the results for the original phase values having a $2 \pi$ range are the best in all cases. In the case of $32 \pi$, which has higher linearity, the compression efficiency is lower than that of $2 \pi$. It can also be seen that the result of full unwrapping without phase limitation is the worst. From this result, it can be confirmed that the increase in the error rate per bit due to the expansion of the dynamic range described above has a greater influence than the linearity of the data.

Phase unwrapping improves the correlation between data by increasing the continuity and linearity of the data. This characteristic makes it possible to expect various possibilities for the application of phase unwrapping. However, the disadvantage of phase unwrapping is that due to phase unwrapping, the data range of $2 \pi$ that the original data had is increased. The increase in data range due to phase unwrapping is proportional to the image size. In the case of applying a process in which loss occurs to a phase component to which phase unwrapping has been applied, it becomes a problem that the dynamic range of data is increased. As the dynamic range increases, the error increases proportionally, so the advantage obtained from the linearity of the data is masked by the error, and the effect of phase unwrapping may disappear. 


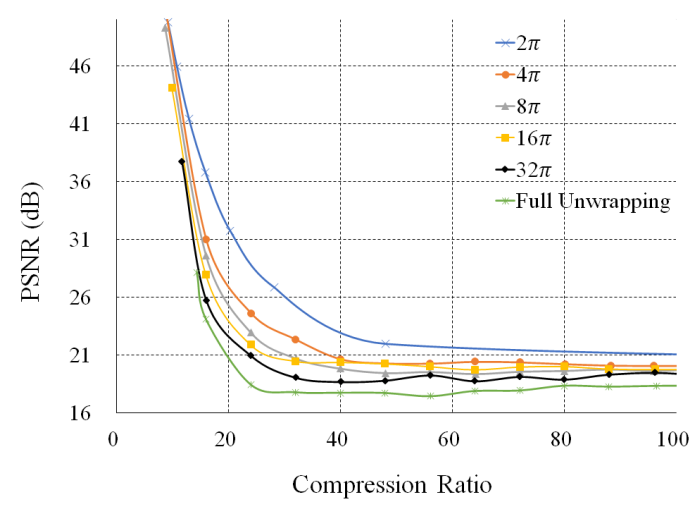

Figure 4. Compression effect of phase unwrapping.

Figure 5 shows the results of the above compression experiment. In this figure, the PSNR of each image is shown along with the image results for both cases, 50:1 and 100:1. In both cases, the PSNR of the phase having a range of $2 \pi$ is $21.99 \mathrm{~dB}$ and $19.88 \mathrm{~dB}$, which is superior to the result of applying phase unwrapping.
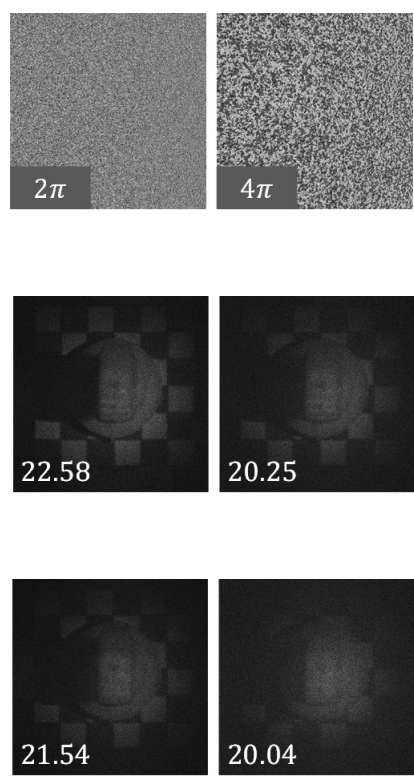

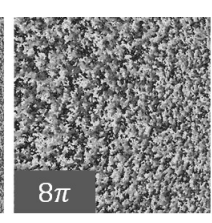

(a)
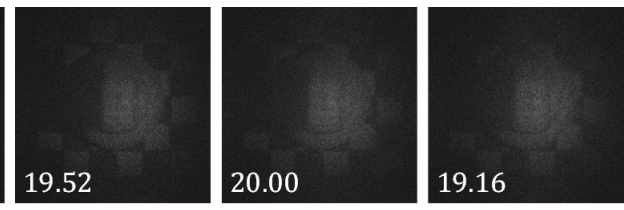

(b)
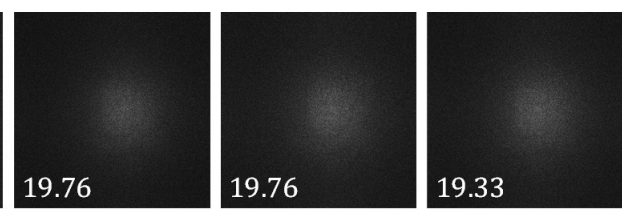

(c)

Figure 5. Coding result using the HEVC codec after applying phase unwrapping and normalization to 8 bits for phase component of a full-complex hologram: (a) unwrapped phase (the value in the subfigure is the dynamic range of phase), (b) reconstruction results after coding phases with 50:1 compression, (c) reconstruction results after coding phases with 100:1 compression (the value is the PSNR).

\section{Phase Compression}

This section describes the proposed phase compression technique. As described above, the full-complex hologram is expressed in terms of amplitude and phase components. Since compressing the amplitude is another research issue, we do not include the compression of the amplitude in the discussion, but focus on compressing the phase. In general, compressing the amplitude may consume a similar effort to compressing the real or imaginary part. 


\subsection{Structure of Codec}

Figure 6 shows the structure of the proposed compression codec. Figure $6 \mathrm{a}$ is the structure of the encoder, and Figure $6 \mathrm{~b}$ is the structure of the decoder. In each figure, the gray part corresponds to the part to compress the phase.

First, we observe the encoding process of Figure 6a. In consideration of the Intra prediction of the HEVC, the segmentation process is performed to divide the hologram into sub-holograms. The separated sub-holograms are unwrapped by considering the direction of the Intra prediction. Unwraped sub-holograms are normalized to an 8-bit width for input to the HEVC. The HEVC can also accept inputs in 10 bits, but we only consider 8-bit inputs to simplify the discussion. Finally, compression is performed using the HEVC.

Next, we observe the decoding process in Figure $6 \mathrm{~b}$. The decoding is the reverse of the encoding process. The result decoded using the HEVC is transformed into an unwrapped phase after de-normalization. The unwrapped phase returns to the original phase values after the rewrapping process. Finally, the decoding result can be obtained by combining the separated sub-holograms.

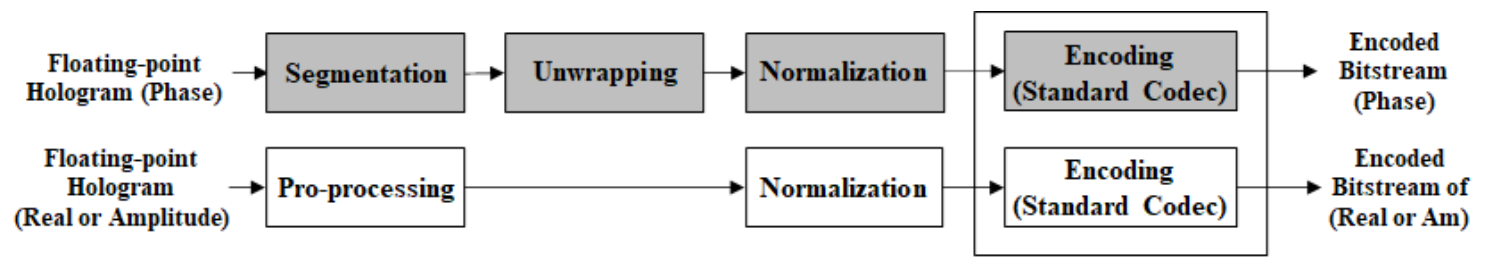

(a)

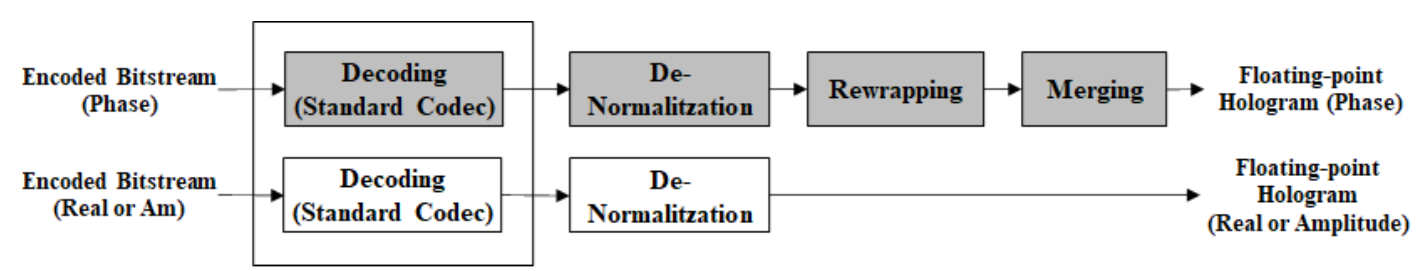

(b)

Figure 6. Proposed structure (a) encoder, (b) decoder. of the hologram codec.

In H.264 / AVC, Intra coding is based on spatial extrapolation of samples from previously decoded image blocks, followed by discrete cosine transform (DCT)-based transform coding [19]. HEVC utilizes the same principle, but further extends it to be able to efficiently represent wider range of textural and structural information in images. The following aspects were considered during the course of HEVC project leading to the selected Intra coding design [19].

HEVC Intra coding is based on predicting the current block $\left(R_{x, y}\right.$ in Figure 7a) by spatially extrapolating the previously decoded left and upper blocks $\left(P_{x, y}\right.$ in Figure 7a). Intra coding of HEVC can support a larger block size than AVC and supports a consistent prediction method for various blocks. In addition, it is possible to efficiently predict not only a structure with a directionality (Angular mode, $N=2 \sim 34$ in Figure $7 \mathrm{~b}$ ) but also a uniform region (Planar and DC mode, $N=0,1$ in Figure $7 \mathrm{~b}$ ). HEVC divides an image into coding units (CUs) for efficient coding, and performs Intra coding in units of prediction units (PUs) having various sizes and shapes from $4 \times 4$ to $32 \times 32$. For residual components generated after Intra prediction, DCT is performed in units of transform units (TU). A boundary smoothing filter can be used to reduce the discontinuity between blocks occurring during Intra prediction [19]. The HEVC supports two special coding modes such as the I_PCM and the transform skipping mode. I_PCM mode is used when efficient coding cannot be performed by other modes. In the I_PCM mode, prediction, transform, quantization, and entropy coding are all bypassed, and predefined bits are allocated. In the transform skipping mode, only transform is skipped. 


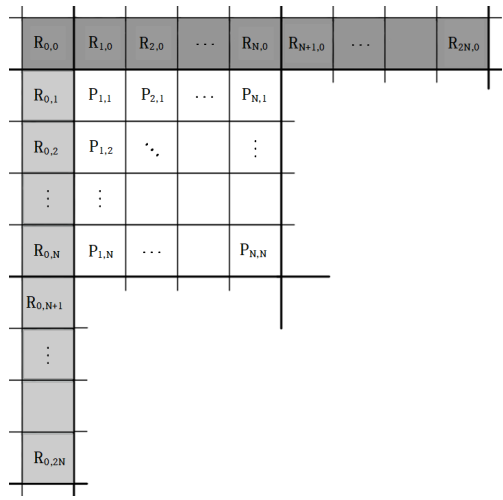

(a)

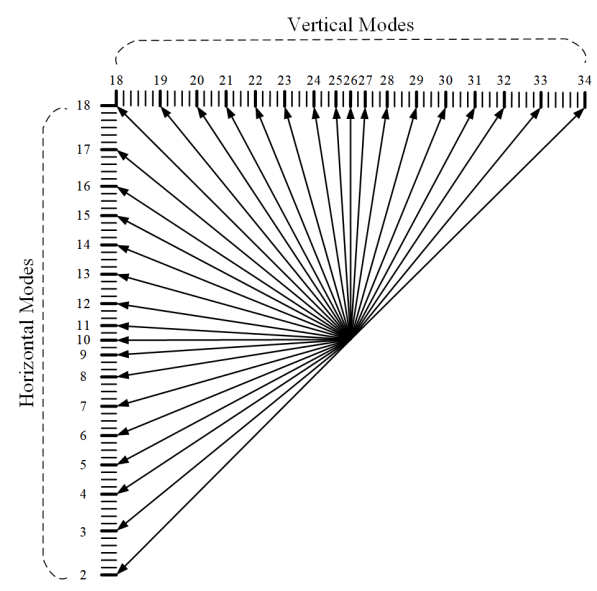

(b)

Figure 7. HEVC Intra coding, (a) reference samples $\mathrm{Rx}, \mathrm{y}$ used in prediction to obtain predicted samples $P_{x, y}$ for a block of size $N \times N$ samples, (b) Figure 2. HEVC angular Intra prediction modes numbered from 2 to 34 and the associated displacement parameters. $\mathrm{H}$ and $\mathrm{V}$ are used to indicate the horizontal and vertical directionalities, respectively, while the numeric part of the identifier refers to the pixels' displacement as 1/32 pixel fractions [19].

We use the HEVC as an anchor codec for compressing the phase information of the still hologram and the Intra prediction of HEVC. Therefore, the phase component of the hologram is transformed into a form suitable for the Intra prediction of the HEVC. As described above, since residual coding is performed on the difference value between $P_{x, y}$ and the original phase after generating $P_{x, y}$ using $R_{x, y}$, the spatial feature of the unwrapped phase must be in a form that is good for predicting $P_{x, y}$ from $R_{x, y}$.

\subsection{Intra Prediction with Unwrapping}

We propose a phase unwrapping technique that can show the highest correlation between the phase component of a hologram and Intra prediction through numerous trials and experiments. This method is simple, but it is a way to overcome the limitation of phase unwrapping for compression shown in Section 2.2. The proposed method is shown in Figure 8a, and the structure of the Intra prediction block we intended is shown in Figure $8 \mathrm{~b} . \quad R$ and $P$ in Figure $8 \mathrm{~b}$ represent the reference block and the prediction block in Figure 7, respectively. Phase linearity increases due to the phase unwrapping technique, but there is a problem in that the error per bit increases in lossy processing such as compression. Therefore, we proposed a method to increase the linearity and decrease the error per bit. That is, it can be regarded as a method that considers the trade-off relationship between linearity and compression efficiency. As shown in the algorithm in Figure $8 \mathrm{a}$, the unwrapping method is limited to the preset threshold $(4 \pi, 8 \pi, 16 \pi$, and $32 \pi)$. This differentiates it from the general unwrapping method, which has an infinite range. Linearity is given by dividing $2 \pi n$ by a certain interval (the value of unwrap in Figure $8 a$ ) and adding it to the original phase.

As described in Section 3.1, the phase unwrapping is applied in consideration of the characteristics of the HEVC Intra coding. In order to simply apply unwrapping and increase the effect of directional prediction in the Intra coding, we propose a modified unwrapping technique in three directions. One object point forms a fringe pattern with a multiple circle shape on the 2D hologram plane. If circle-shaped fringe patterns with the same shape in all directions overlap very much in the case of multiple object points, it may be predicted that the resulting pattern does not have a dominant directionality in any particular direction. Figure 9 shows the results of measuring the HEVC Intra mode while rotating the hologram around the center. The graph in Figure 9 shows that the angular directions in the HEVC Intra prediction are variously distributed within 
the hologram. In the graph of Figure 9, the angular direction number is the same as Figure $7 \mathrm{~b}$. We propose an algorithm that manipulates the hologram using the modified phase unwrapping so that HEVC Intra prediction can be performed well. Therefore, it is possible to select some of the angular modes used in the Intra prediction. We select the vertical, horizontal, and diagonal directions corresponding to 0 degrees (180 degrees), 45 degrees (225 degrees), and 90 degrees (270 degrees), and then manipulate the hologram using a modified unwrapping technique.

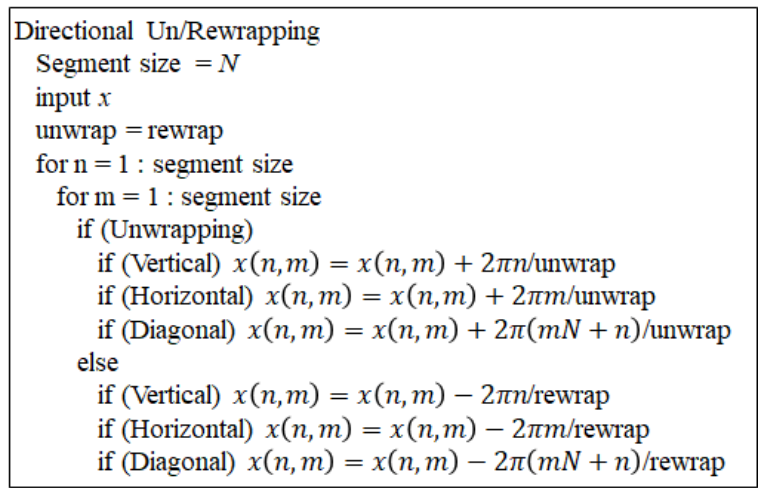

(a)

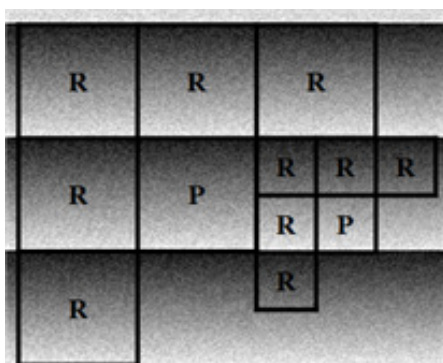

(b)

Figure 8. Proposed unwrapping algorithm (a) the pseudo-code, (b) the segments for the intra prediction.

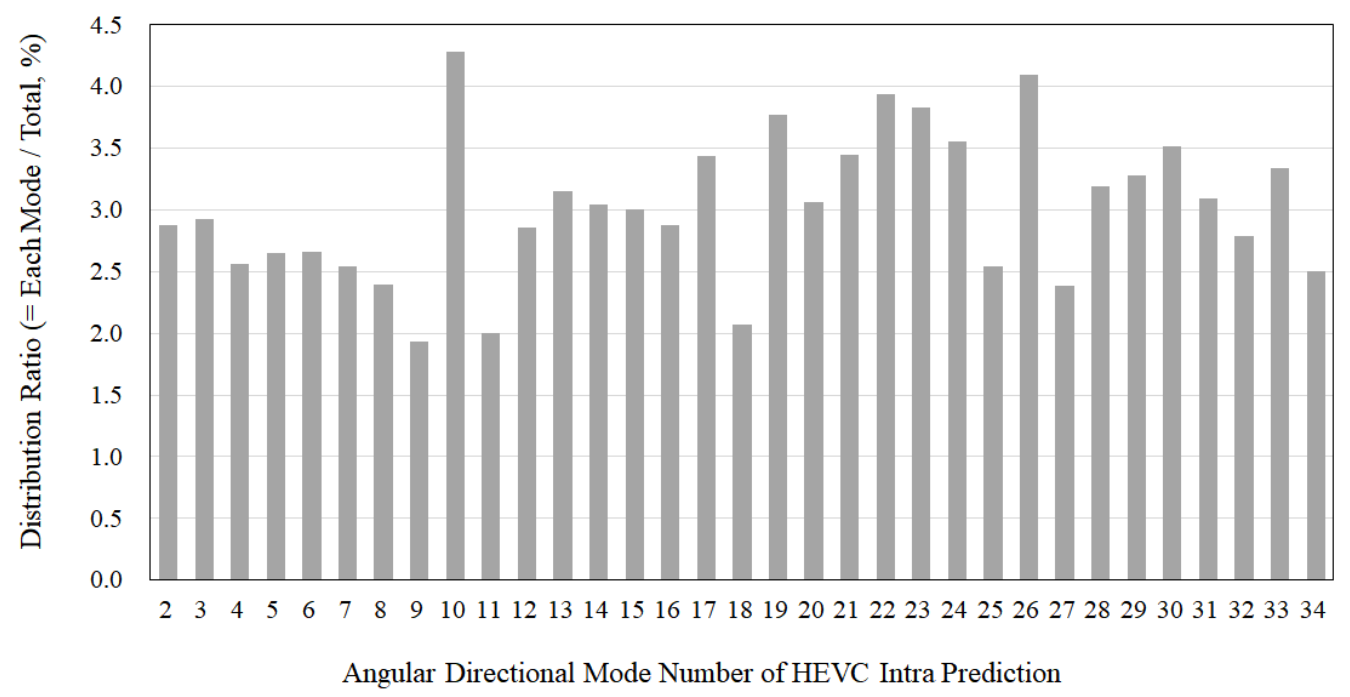

Figure 9. Distribution of angular directional modes.

The three modified unwrapping methods are shown in Figure 10. Figure 10a-c show how to unwrap the phase in vertical, horizontal, and diagonal directions, respectively. The unwrapping proceeds from the dark to the bright region. That is, the brightness can be viewed as the magnitude of the dynamic range of the unwrapped phase. Each figure in Figure 10 consists of a total of 16 blocks. Each block corresponds to a CU in the HEVC codec. Considering HEVC Intra prediction, we divide the block as shown in Figure 10 to the same size as the CU used for Intra prediction, and apply phase unwrapping. 


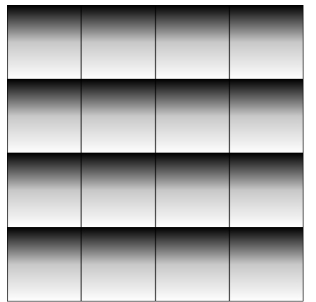

(a)

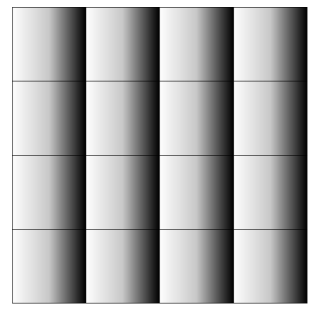

(b)

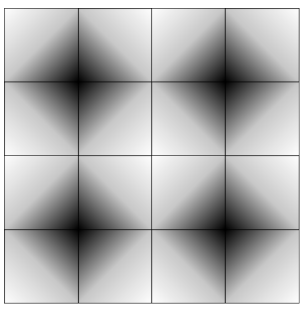

(c)

Figure 10. Proposed modified unwrapping methods considering HEVC Intra prediction for (a) vertical, (b) horizontal, and (c) diagonal prediction.

\section{Experiment and Result}

In this section, the experimental results of the proposed phase compression scheme are shown, and it is shown that the proposed scheme has superior performance compared to the scheme in which phase is compressed only by the HEVC.

\subsection{Environment}

The proposed algorithm was implemented using $\mathrm{C} / \mathrm{C}++$, OpenCV, OpenMP, and CUDA in Intel I7-7700K CPU @3.6 GHz, 64 GB RAM, and 64-bit Window10 environment. We used the full-complex holograms published by JPEG Pleno [5].

Table 2 summarizes the conditions for compression experiments. We divided the hologram into three sizes $(64 \times 64,32 \times 32$, and $16 \times 16)$, and each segment is compressed under conditions of various CU sizes. A total of 4 dynamic ranges for unwrapping ( $4 \pi$, $8 \pi, 16 \pi$, and $32 \pi)$ were tested. The phase was compressed using the HEVC Intra and the JPEG2000, and the proposed method was compressed using the HEVC Intra.

Table 2. Coding conditions for the experiment.

\begin{tabular}{|c|c|c|c|}
\hline Segment Size & CU Size & Dynamic Range of Unwrapping & Codec \\
\hline \multirow{3}{*}{$64 \times 64$} & 64 & \multirow{6}{*}{$4 \pi, 8 \pi, 16 \pi, 32 \pi$} & \multirow{6}{*}{ HEVC Intra, JPEG2000 } \\
\hline & 32 & & \\
\hline & 16 & & \\
\hline \multirow{2}{*}{$32 \times 32$} & 32 & & \\
\hline & 16 & & \\
\hline $16 \times 16$ & 16 & & \\
\hline
\end{tabular}

\subsection{Unwrapping Result}

In this section, some unwrapping results are shown. Figure 11 shows the result of full unwrapping for the hologram used in the experiment and the result of unwrapping using the proposed method.

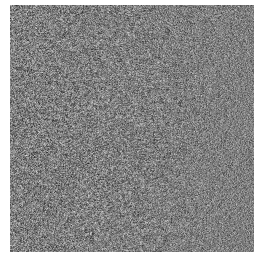

(a)

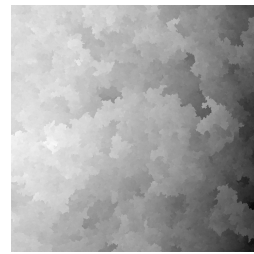

(b)

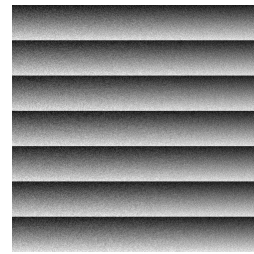

(c)

Figure 11. Result of the proposed phase unwrapping: (a) original phase, (b) full unwrapping, (c) proposed vertical phase unwrapping. 
Figure 12 shows the unwrapping results for each direction. In each figure, it corresponds to the vertical, horizontal, and diagonal unwrapping in order from the top. Each figure is the result of different dynamic range. If the dynamic range is large, the linearity of the phase increases, and the error rate per bit increases as the amount of information per bit increases after normalization.
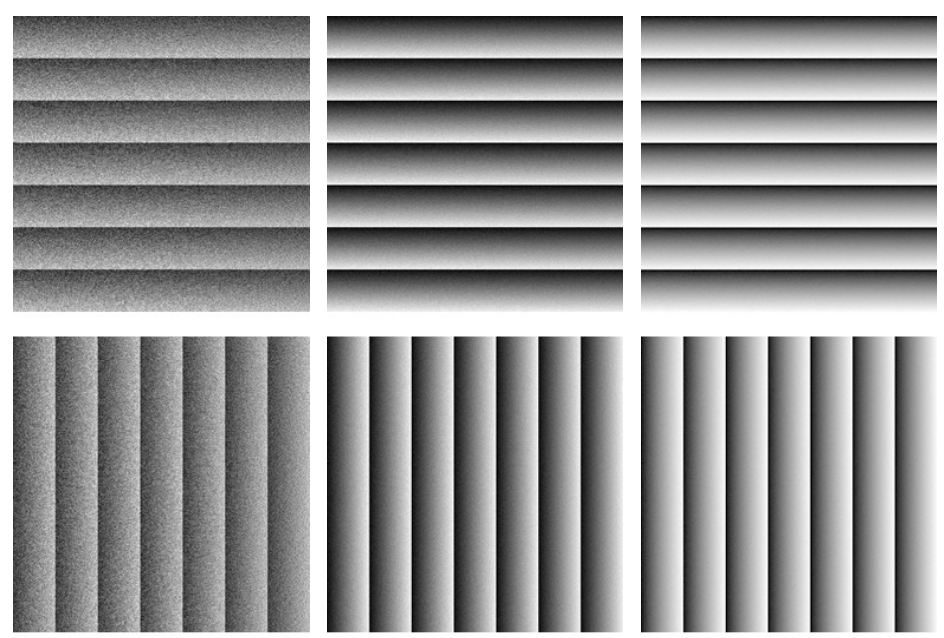

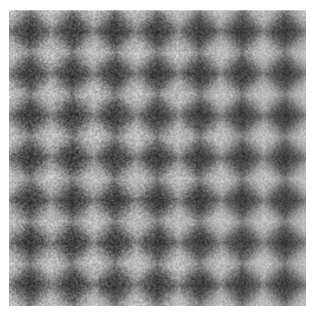

(a)

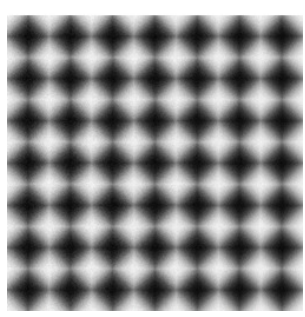

(b)

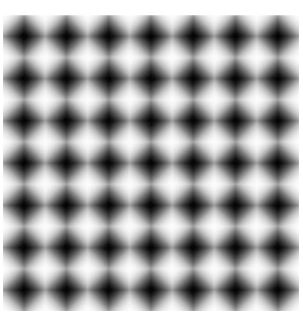

(c)

Figure 12. Result of the proposed phase unwrapping for limiting dynamic range to (a) $4 \pi$, (b) $16 \pi$ and (c) $32 \pi$ (In each subfigure, the first row is the vertical unwrapping, the second is the horizontal unwrapping, and the last is the diagonal unwrapping).

\subsection{Coding Result}

In this section, we show the compression and decompression results as graphs expressed in compression ratio and PSNR. We performed experiments for all combinations of conditions shown in Table 2, and selected only the best results among them and plotted them as a graph. According to the unwrapping direction, the PSNR results according to the compression ratio from Figures 13-15 are plotted as a graph. Each figure shows the quality of the phase and the quality of the reconstruction result according to the compression rate. We used the original amplitude in order to accurately observe the compression effect of the phase. Each graph must include the dynamic range of $4 \pi, 8 \pi, 16 \pi$, and $32 \pi$, and the segment and the size of the $\mathrm{CU}$ were selected under conditions that showed the best results.

Figures 13-15 are compression results for the unwrapped phase in the vertical, horizontal, and diagronal directions, respectively. Although there are some differences in quantitative results, it can be seen that the PSNR of the hologram and the reconstruction according to the compression ratio for each dynamic range are almost similar.

As can be seen from the experimental results of Figure 4, the same trend (a phenomenon in which the error per bit increases and the compression efficiency decreases as the dynamic range increases) occurred in the results of Figures 13-15. The results for the case of $32 \pi$ show poor quality at most compression rates. However, unlike the results in Figure 4, there is no significant difference in compression efficiency according to each dynamic range. This can be interpreted as proving that unwrapping improves the compression efficiency as a whole. 
In the results of Figure 13 , it can be seen that compression efficiency is generally high under the condition that the segment size is $64 \times 64$ and the $\mathrm{CU}$ is $32 \times 32$.

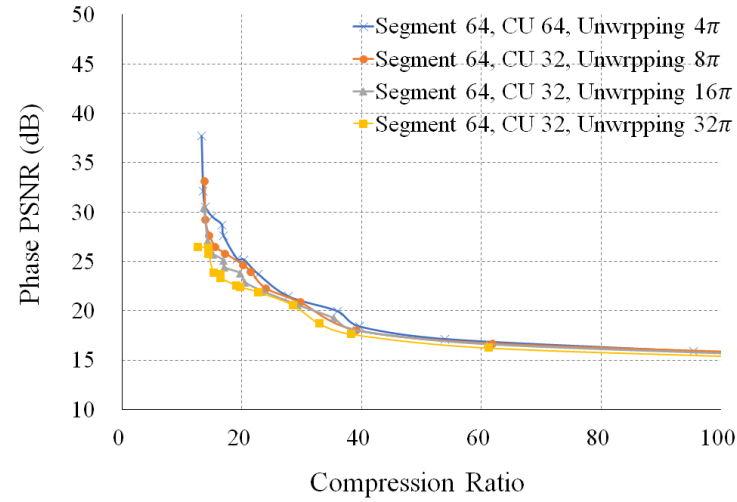

(a)

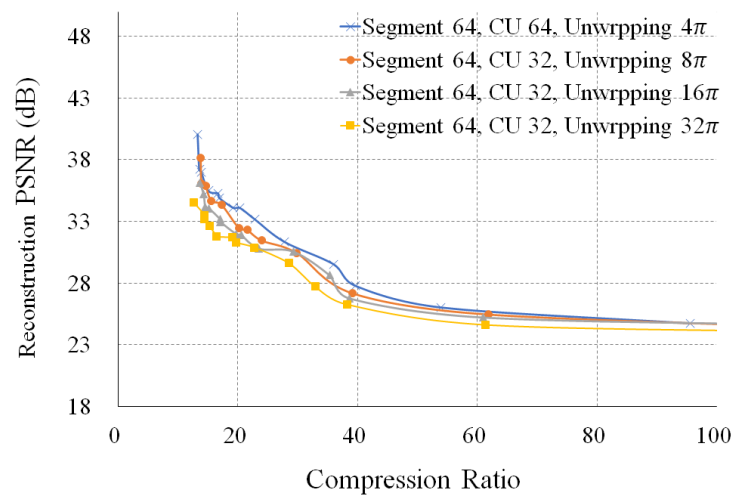

(b)

Figure 13. Compression results with the vertical unwrapping: (a) phase, (b) reconstruction result.

In the results of Figure 14, it can be seen that compression efficiency is generally high under the condition that the segment size is $64 \times 64$ and the CU size is $64 \times 64$. The compression result using the horizontal unwrapping was superior to the vertical unwrapping result in the condition of the larger CU size. In the case of the horizontal unwrapping, unlike other cases, the difference in reconstruction results is very small.

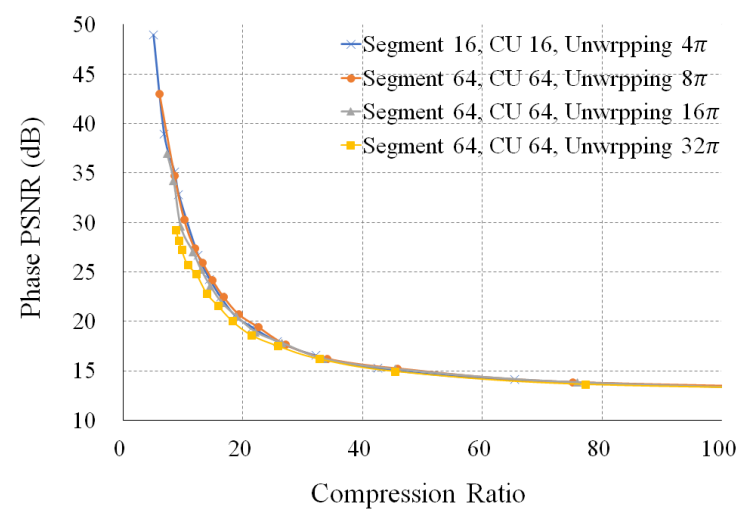

(a)

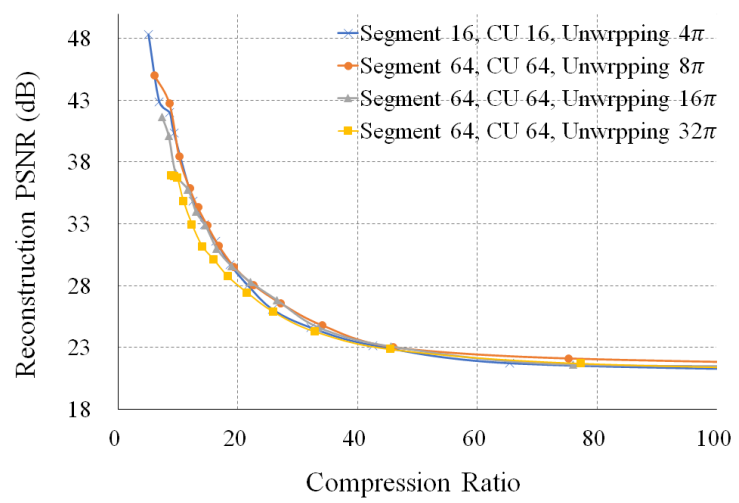

(b)

Figure 14. Compression results with the horizontal unwrapping: (a) phase, (b) reconstruction result.

In the result of Figure 15, it can be seen that compression efficiency is high in both the segment and the CU size are $64 \times 64$. Overall, the quality according to the compression ratio is almost the same as in other cases.

Figure 16 plots the results of the best performance condition among each method. In addition, the result of phase compression using the HEVC Intra and the JPEG2000 without applying unwrapping was plotted together.

Figure 16a shows the phase compression results, and Figure 15b shows the restoration results. Figure $16 \mathrm{c}, \mathrm{d}$ are the conversion of the phase compression results into real and imaginary parts, respectively. In Figure 16a,b, compression efficiency is excellent when the vertical unwrapping method is used in most cases. The degree of improvement of the compression performance varies depending on the compression ratio, but the proposed method shows better characteristics as the compression ratio increases. The JPEG2000 has the lowest overall performance in high compression, but some good performance in low compression. At a compression ratio of 50:1 or higher, all proposed schemes showed better performance than the case of using the HEVC Intra and the JPEG2000. At a compression 
ratio of 100:1, the vertical unwrapping method shows better performance over $2 \mathrm{~dB}$ in phase and reconstruction results than other methods. Compared to the JPEG2000 and the HEVC Intra, our method shows a difference of almost $4 \mathrm{~dB}$ in all of the phase and reconstruction results. In the results of real and imaginary parts, shown in Figure 16c,d, this trend is almost the same. In particular, in the case of using the proposed method, it can be seen that the PSNR of the real part and the imaginary part are kept almost similar.

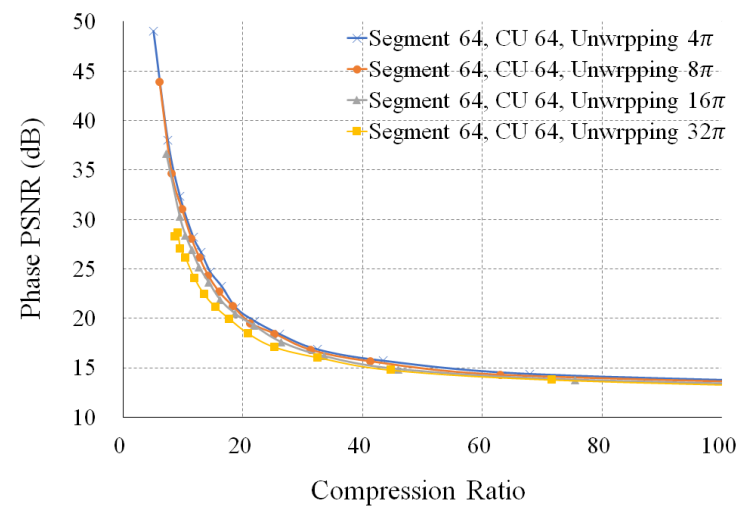

(a)

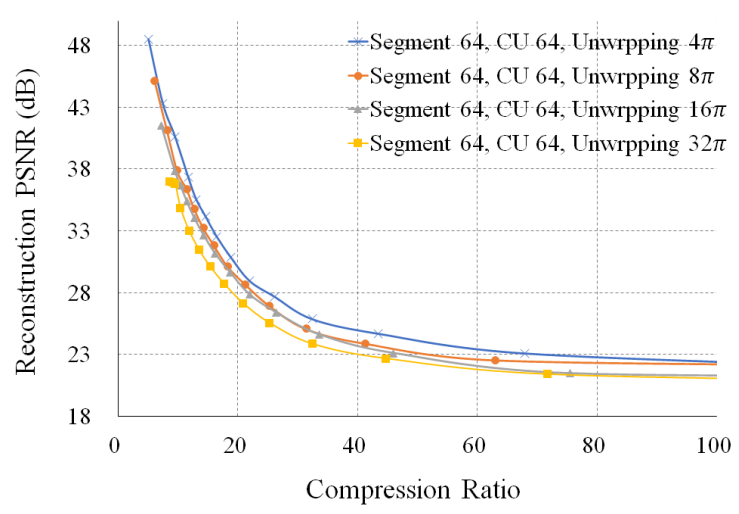

(b)

Figure 15. Compression results with the diagonal unwrapping: (a) phase, (b) reconstruction result.

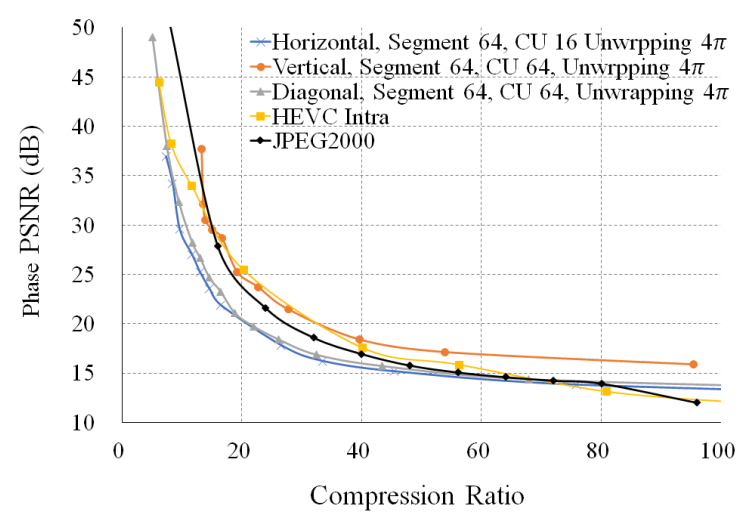

(a)

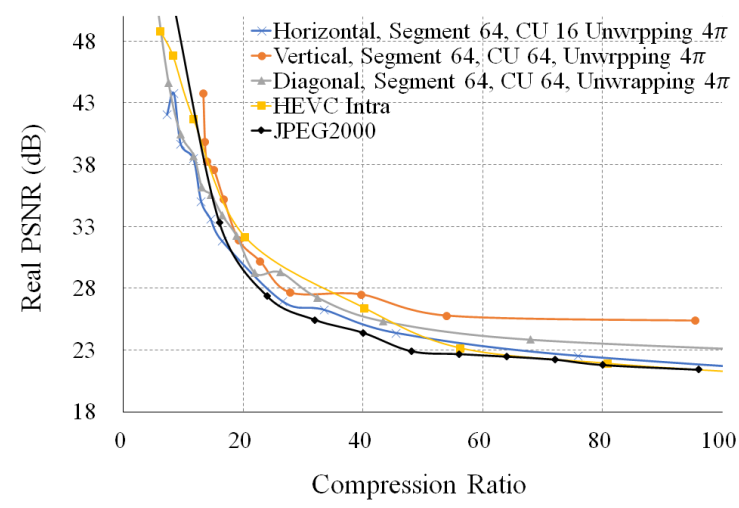

(c)

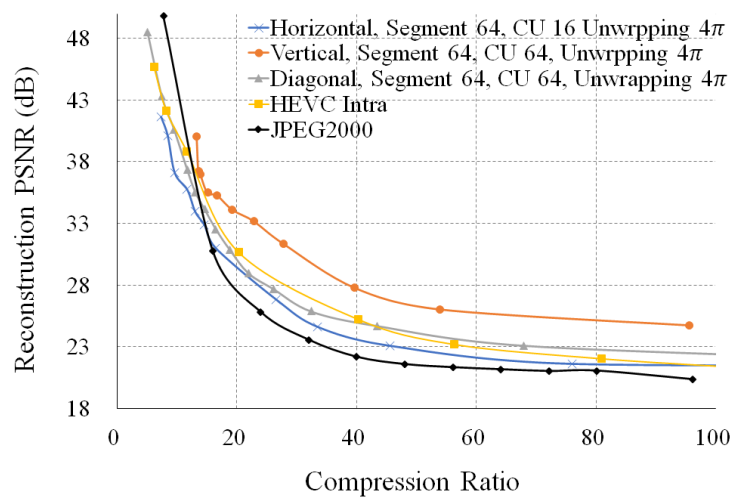

(b)

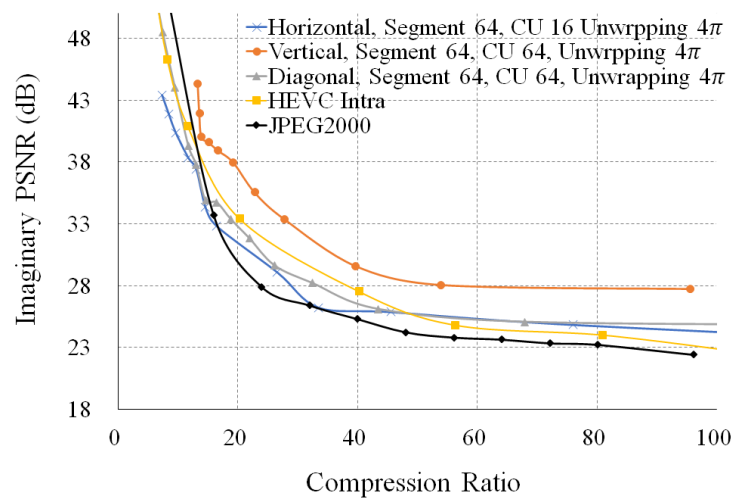

(d)

Figure 16. Performance comparison of the various methods: (a) phase, (b) reconstruction, (c) real, and (d) imaginary part.

The hologram phase information may vary depending on the hologram generation method and the amount of random phase. For these various phase information, the segment and CU size having the most optimal compression efficiency may differ. After setting 
the segment and the CU size in advance within the size range dependent on the CU size of HEVC, the greedy method that obtains the best compression efficiency result after coding the phase information under various conditions can be applied.

The numerical reconstruction result of the phase information is shown as an image. We performed experiments using holograms provided by JPEG Pleno. Among them, the resulting images for the Horse [18] and Dices [20,21] holograms are shown in Figures 17-19. Figure 17 is the reconstruction result of the original hologram. The resolution of Horse, which is the Fresnel hologram method, is $972 \times 972$ and the pixel pitch is $6.4 \mu \mathrm{m}$. It has only the red channel of $632.8 \mathrm{~nm}$ wavelength, and the reconstruction distance is $14 \mathrm{~cm}$. Dices, which is an angular spectrum method, has a size of $1920 \times 1080$ and a pixel pitch of $6.4 \mu \mathrm{m}$. The wavelengths for the red, green, and blue channels are 640,532, and $483 \mathrm{~nm}$, respectively. Its reconstruction distance is $0.327 \mathrm{~cm}$.

The reconstruction for the Fresnel diffraction method is defined by Equation (1). $\Delta x$ and $\Delta y$ are sampling periods for the $x$-axis and $y$-axis, respectively, and the number of samplings is $\mathrm{M}$ and $\mathrm{N} . H(p, q)$ represents a hologram, and $k_{0}$ represents a wave number. $(m, n)$ and $(p, q)$ are indexes of samples in the spatial domain and Fourier domain, respectively.

$$
O(p, q)=\frac{j k_{o} e^{-j k_{o} z}}{2 \pi z} \exp \left\{-j \pi \lambda z\left[\left(\frac{p}{M \Delta_{x}}\right)^{2}+\left(\frac{q}{N \Delta_{y}}\right)^{2}\right]\right\} \times \operatorname{IDFT}_{2 D}\left\{H(m, n) \exp \left[\frac{-j k_{o}}{2 z}\left(m^{2} \Delta_{x}^{2}+n^{2} \Delta_{y}^{2}\right)\right]\right\}
$$

The reconstruction for the angular spectrum method is defined by Equation (2). $k_{x}$ and $k_{y}$ are spatial radian frequencies, respectively. Since $\Delta_{k x}$ and $\Delta_{k y}$ are defined as frequency resolution, $\Delta_{k x}=2 \pi / M \Delta_{x}$ and $\Delta_{k y}=2 \pi / M \Delta_{y}$. Equations (1) and (2) are described in detail in [22].

$$
O(p, q)=D F T_{2 D}\left[\operatorname{IDFT}_{2 D} H(m, n) * \exp \left[-j k_{o} z \sqrt{1-\frac{\left(p \Delta_{k x}\right)^{2}}{k_{o}^{2}}-\frac{\left(q \Delta_{k y}\right)^{2}}{k_{o}^{2}}}\right]\right]
$$

Figure 18 is for the Horse hologram, and Figure 19 is for the Dices hologram. In Figures 18 and 19, each subfigure includes the result of the JPEG2000, the HEVC Intra, and the proposed method. In both figures, (a) and (b) are reconstruction images for compression ratios of 50:1 and 100:1, respectively. At a compression ratio of 50:1 in the two images, it is difficult to visually confirm the difference in quality. In Figure 18, it can be clearly seen that the proposed method at a compression ratio of 100:1 shows better visual quality than other methods. In Figure 19, it can be seen that the color is deteriorated in the compression result by JPEG2000. From the 100:1 compression ratio in Figure 19, it can be seen that the proposed method has subtly superior visual quality compared to other methods.

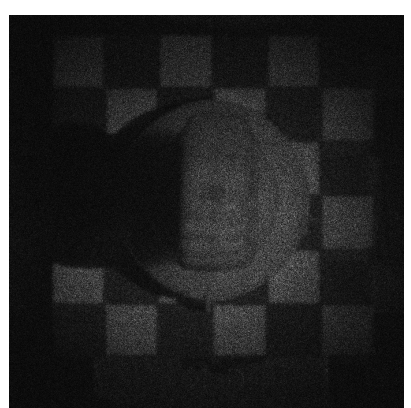

(a)

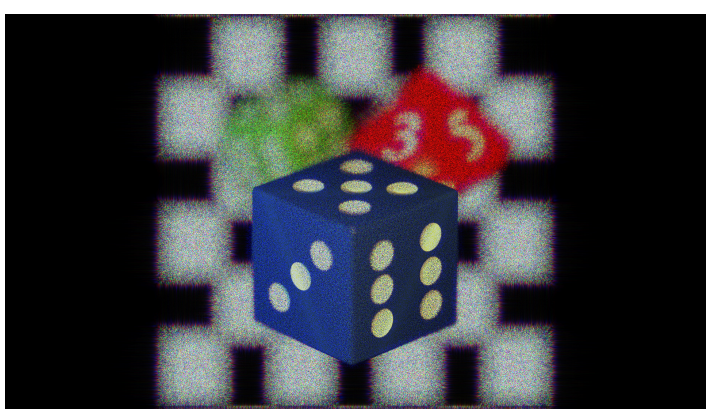

(b)

Figure 17. Numerical reconstruction image of the original hologram: (a) Horse [18], (b) Dices [20,21]. 

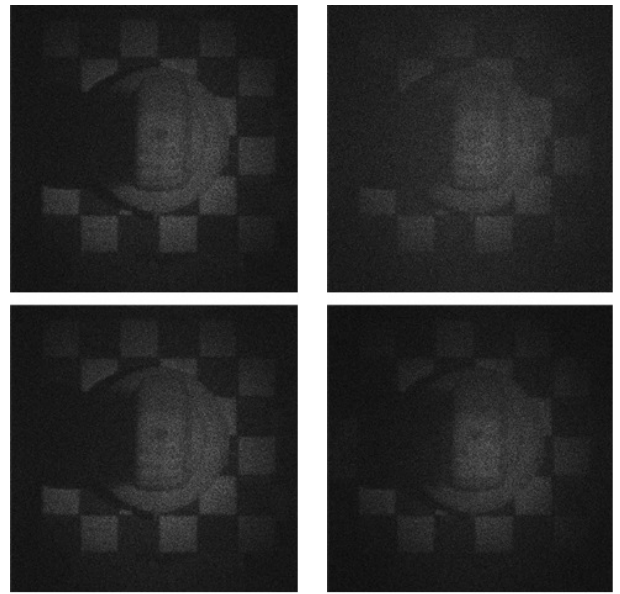

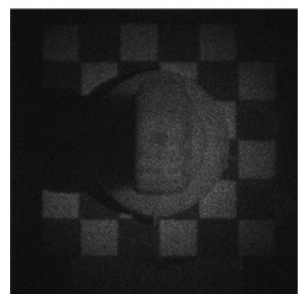

(a)

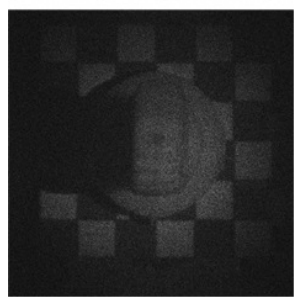

(b)

Figure 18. Reconstruction result after compression of the phase information of the Horse hologram [18]: (a) 50:1, (b) 100:1 (JPEG2000, HEVC Intra, the proposed method from the top of each figure).

The calculation time for each compression method was measured and summarized in Table 3. Similar to general image compression, it takes a long time in low compression and a short time in high compression. Since JPEG2000 is a relatively simple codec compared to HEVC Intra, the operation time is very short. Comparing the HEVC Intra with our method, our method takes a shorter time than the case of compressing with HEVC Intra because the phase is converted into a form that is easy to compress using the modified unwrapping method.
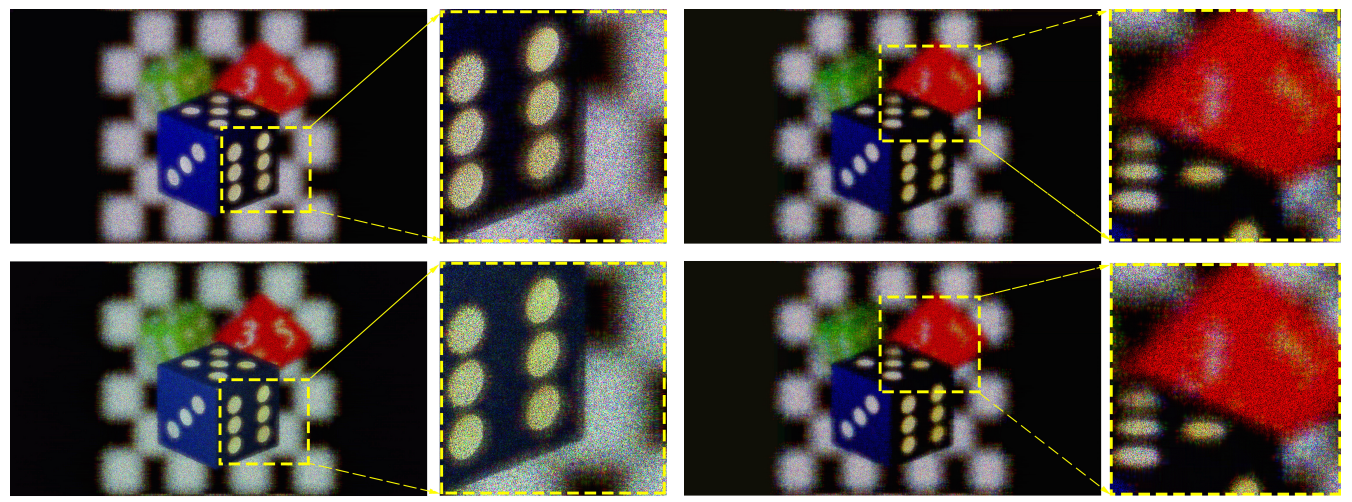

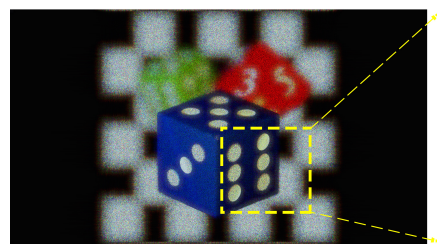

(a)
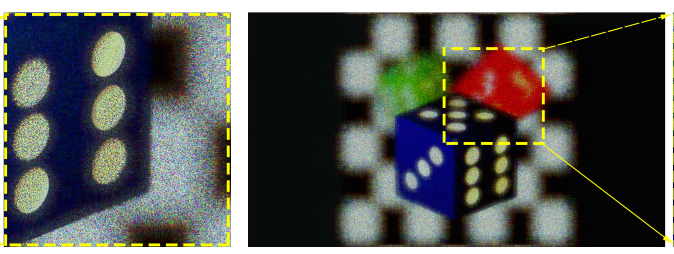

(b)

Figure 19. Reconstruction result after compression of the phase information of the Dices hologram [20,21]: (a) 50:1, (b) 100:1 (JPEG2000, HEVC Intra, the proposed method from the top of each figure). 
Table 3. Computation time for compression ratio.

\begin{tabular}{cccc}
\hline & \multicolumn{3}{c}{ Computation Time [s] } \\
\cline { 2 - 4 } & $\mathbf{1 0 : 1}$ & $\mathbf{5 0 : 1}$ & $\mathbf{1 0 0 : 1}$ \\
\hline JPEG2000 & 1.55 & 1.12 & 0.99 \\
\hline HEVC Intra & 30.28 & 25.32 & 21.98 \\
\hline Ours & 16.21 & 15.54 & 15.12 \\
\hline
\end{tabular}

\section{Conclusions}

In this paper, a method for compressing the phase of a full-complex hologram is proposed. We tried a method to directly compress the phase information to overcome the drawbacks of the method of independently compressing real and imaginary parts. We selected the HEVC as the anchor codec and proposed a modified unwrapping algorithm with three directions to transform it into phase information in a form suitable for the HEVC Intra prediction. The unwrapping algorithm is configured to be optimized for the HEVC Intra prediction. In order to synchronize unwrapping with the Intra prediction, the size of the sub-hologram was selected and the size of the $\mathrm{CU}$ was adjusted. Compared with the result of compressing the phase using the HEVC, the hologram was improved by $2 \mathrm{~dB}$ or more and the reconstruction result was improved by more than $4 \mathrm{~dB}$ at a compression ratio of 100:1. In the standardization of JPEG Pleno, we want to provide an opportunity to reconsider the possibility of compressing the amplitude and phase of the full-complex hologram rather than the real and imaginary form.

Author Contributions: Conceptualization, J.-K.K. and Y.-H.S.; methodology, J.-K.K.; software, K.-J.O. and J.-W.K.; validation, Y.-H.S.; writing-review and editing, Y.-H.S. and D.-W.K.; visualization, Y.-H.S. and D.-W.K.; supervision, Y.-H.S., K.-J.O. and J.-W.K.; funding acquisition. All authors have read and agreed to the published version of the manuscript

Funding: The present research has been conducted by the Research Grant of Kwangwoon University in 2021. This work was supported by Giga KOREA project, [GK20D0100, Development of Telecommunications Terminal with Digital Holographic Table-top Display].

Institutional Review Board Statement: Not applicable.

Informed Consent Statement: Not applicable.

Data Availability Statement: Not applicable.

Conflicts of Interest: The authors declare no conflict of interest.

\section{References}

1. Gabor, D. A new microscopic principle. Nature 1948, 161, 777-778. [CrossRef] [PubMed]

2. Hariharan, P. Basics of Holography; Cambridge University Press: Cambridge, UK, 2002.

3. Osten, W.; Faridian, A.; Gao, P.; Körner, K.; Naik, D.; Pedrini, G.; Singh, A.K.; Takeda, M.; Wilke, M. Recent advances in digital holography [Invited]. Appl. Opt. 2014, 53, G44-G63. [CrossRef] [PubMed]

4. Yoshikawa, H. Digital holographic signal processing. In Proceedings of the TAO First International Symposium on Three Dimensional Image Communication Technologies, Tokyo, Japan, 6-7 December 1993; pp. S-4-2.

5. JPEG Pleno. Available online: https://jpeg.org/jpegpleno/ (accessed on 1 January 2021).

6. Naughton, T.J.; Frauel, Y.; Javidi, B.; Tajahuerce, E. Compression of digital holograms for three-dimensional object reconstruction and recognition. Appl. Opt. 2002, 41, 4124-4132. [CrossRef] [PubMed]

7. Naughton, T.J.; Frauel, Y.; Matoba, O.; Bertaux, N.; Tajahuerce, E.; Javidi, B. Three-dimensional imaging, compression, and reconstruction of digital holograms. In Opto-Ireland 2002: Optical Metrology, Imaging, and Machine Vision; Shearer, A., Murtagh, F.D., Mahon, J., Whelan, P.F., Eds.; International Society for Optics and Photonics, SPIE: Bellingham WA, USA, 2003; Volume 4877, pp. 104-114. [CrossRef]

8. Yoshikawa, H.; Sasaki, K. Image scaling for electroholographic display. In Practical Holography VIII; Benton, S.A., Ed.; International Society for Optics and Photonics, SPIE: Bellingham WA, USA, 1994; Volume 2176, pp. 12-22. [CrossRef] 
9. Cheremkhin, P.A.; Kurbatova, E.A. Numerical comparison of scalar and vector methods of digital hologram compression. In Holography, Diffractive Optics, and Applications VII; Sheng, Y., Yu, C., Zhou, C., Eds.; International Society for Optics and Photonics, SPIE: Bellingham WA, USA, 2016; Volume 10022, pp. 455-464. [CrossRef]

10. Ahar, A.; Blinder, D.; Bruylants, R.; Schretter, C; Munteanu, A.; Schelkens, P. Subjective quality assessment of numerically reconstructed compressed holograms. Proc. SPIE 2015, 95990K, 1-15. doi :10.1117/12.2189887.

11. Peixeiro, J.; Brites, C.; Ascenso, J.; Pereira, F. Digital holography: Benchmarking coding standards and representation formats. In Proceedings of the 2016 IEEE International Conference on Multimedia and Expo (ICME), Seattle, WA, USA, 11-15 July 2016; pp. 1-6. [CrossRef]

12. Peixeiro, J.P.; Brites, C.; Ascenso, J.; Pereira, F. Holographic Data Coding: Benchmarking and Extending HEVC With Adapted Transforms. IEEE Trans. Multimed. 2018, 20, 282-297. [CrossRef]

13. Darakis, E.; Soraghan, J.J. Use of Fresnelets for Phase-Shifting Digital Hologram Compression. IEEE Trans. Image Process. 2006, 15, 3804-3811. [CrossRef] [PubMed]

14. Darakis, E.; Soraghan, J.J. Reconstruction domain compression of phase-shifting digital holograms. Appl. Opt. 2007, 46, 351-356. [CrossRef] [PubMed]

15. Darakis, E.; Naughton, T.J.; Soraghan, J.J. Compression defects in different reconstructions from phase-shifting digital holographic data. Appl. Opt. 2007, 46, 4579-4586. [CrossRef] [PubMed]

16. Sim, J.Y. Digital Hologram Compression Using Correlation of Reconstructed Object Images. In Advances in Image and Video Technology; Ho, Y.S., Ed.; Springer: Berlin/Heidelberg, Germany, 2012; pp. 204-214.

17. Abdul-Rahman, H.S.; Gdeisat, M.A.; Burton, D.R.; Lalor, M.J.; Lilley, F.; Moore, C.J. Fast and robust three-dimensional best path phase unwrapping algorithm. Appl. Opt. 2007, 46, 6623-6635. [CrossRef] [PubMed]

18. Bernardo, M.V.; Fernandes, P.; Arrifano, A.; Antonini, M.; Fonseca, E.; Fiadeiro, P.T.; Pinheiro, A.M.; Pereira, M. Holographic representation: Hologram plane vs. object plane. Signal Process. Image Commun. 2018, 68, 193-206. [CrossRef]

19. Lainema, J.; Bossen, F.; Han, W.; Min, J.; Ugur, K. Intra Coding of the HEVC Standard. IEEE Trans. Circuits Syst. Video Technol. 2012, 22, 1792-1801. [CrossRef]

20. Gilles, A.; Gioia, P.; Cozot, R.; Morin, L. Computer generated hologram from Multiview-plus-Depth data considering specular reflections. In Proceedings of the 2016 IEEE International Conference on Multimedia Expo Workshops (ICMEW), Seattle, WA, USA, 11-15 July 2016; pp. 1-6. [CrossRef]

21. Gilles, A.; Gioia, P.; Cozot, R.; Morin, L. Hybrid approach for fast occlusion processing in computer-generated hologram calculation. Appl. Opt. 2016, 55, 5459-5470. [CrossRef] [PubMed]

22. Dwivedi, G.; Sharma, A.; Debnath, S.; Rajkumar. Comparison of numerical reconstruction of digital holograms using angular spectrum method and Fresnel diffraction method. J. Opt. 2017. [CrossRef] 